Oeber Entzündung des vorderen Abschnittes der Chorioidea (des Ciliarkörpers) als Nachkrankheit der febris recurrens.

Von

Dr. Logetschnikow in Moskau.

(Vortrag, gehalten in der medicinischen Section der zweiten Versammlung Russischer Naturforscher zu Moskau, den 29. Aug. 1869 von S. Log et schnikow, Assistenzarzt in der Augenheilanstalt zu Moskau.)

\title{
Meine Herren!
}

Es sei mir vergönnt, Ihre Aufmerksamkeit auf eine Augenkrankheit zu lenken, die in mehreren Beziehungen und hauptsächlich in Beziehung ihres räthselhaften Verhältnisses zur febris recurrens ein nicht geringes Interesse darbietet. Schon im Jahre 1826, während und nach der Epidemie der febris recurens in Dublin, wurde von den englischen Aerzten ein eigenthümliches Augenleiden beobachtet und dasselbe in Zusammenhang mit der febris recurrens gebracht. Von den damaligen Beschreibungen dieser Augenaffection kann die von Wallace als die zutreffendste genannt werden; allein beim gegenwärtigen Stande der Ophthalmologie erscheint sie freilich als bei weitem ungenügend und, was die Localisation des Uebels anbetrifft, vollkommen irrthümlich. Eine neue Epidemie im Jahre 1843 in Schottland gab wiederum Gelegenheit 
dieselbe Augenaffection zu treffen und deren Beschreibung verdanken wir Mackenzie.*) Der von ihm geschilderte Symptomencomplex lässt für die damalige vorophthalmoscopische Zeit an Klarheit und - ich möchte sagen - an Vollständigkeit nichts zu wünschen übrig. Bei der Würdigung der Symptome, bei deren Localisation kommt aber auch dieser hervorragende Ophthalmologe zu der irrigen Auffassung, dass das Wesen der Krankheit in der Netzhaut mit nachfolgenden Veränderungen der Iris, der Sclera, zum Theil der Chorioidea und in der Mehrzahl auch der übrigen Augenhäute - daher der Name „Ophthalmitis" i. e. Entzündung des gesammten Bulbus - zu suchen sei.

Eine weit verbreitete Epidemie der febris recurrens, die seit dem Jahre 1865 zuerst in. Petersburg und bald darauf in Moskau auftrat, gab zum drittenmal Veranlassung sich mit der von englischen Aerzten beschriebenen und von Andern (wie Middlemore, Himly) in vollkommenes Dunkel gehüllten Augenkrankheit zu beschäftigen, sie näher in's Auge zu fassen und exacter $z u$ würdigen, da wir indessen eine ganze Reihe neuer diagnostischer Hülfsmittel - in der Ophthalmoscopie, feinern Bestimmung der Sehfähigkeit, der Refractions und Accommodationsanomalien - kennen gelernt hatten; und war ich so glücklich im Jahre 1866 in einer der Sitzungen hiesiger physicalisch-medicinischen Gesellschaft die Resultate meiner ersten, aus achtzig Fällen entnommenen Beobachtungen niederlegen zu können.**)

Das Wesen der Krankheit suchte ich in der primären Entzündung des vordern Abschnitts der Aderhaut, des

*) Mackenzie. Traité pratique des maladies de l'oeuil, traduit par Wallomont et Testelin. Paris 1857. Tome II. pag. 102-112.

**) Siehe: Sitzungsberichte der physicaliseh-medioinischen Gesellscbaft zu Moskau, 30. Mai 1866, auch Moskauer medioinische Zeitung Nr. 44, 1866 (russisch). 
Ciliarkörpers, mit einer zwar sehr häufigen, aber durchaus nicht nothwendigen, secundären Erkrankung der Iris. Glaskörperopacitäten hielt ich für das wesentlichste und constanteste Symptom, dagegen war die Theilnahme (wenigstens eine nahmhafte) seitens der Retina, Sclera und Conjunctiva aus dem Krankheitsbilde ausgeschlossen.

Aus einer Mittheilung von Blessig, die er im Jahre 1867 dem internationalen Congresse der Ophthalmologen zu Paris machte, ersieht man, dass auch in Petersburg die Epidemie der febris recurrenz eine Augenkrankheit zur Nachfolge hatte. Gegen die Annahme einer Cyklitis stellt Blessig die Diagnose einer Iridochorioiditis auf und sagt: „une cyklite, dans le sens ordinaire, n'existait jamais." Die erste Periode, wo die Augenaffection blos durch Accommodationsbeschwerden und sodann durch Glaskörperopacitäten sich kennzeichnet, scheint ihm entgangen zu sein, da er nur ausgesprochene Entzündungen gesehen und beschrieben hat, daher er auch die amblyopische Periode von $\dot{W}$ allace in $\mathbf{A b}$ rede stellt.*)

Seit meiner ersten Mittheilung ist die Anzahl der Fälle, wo der Zusammenhang der Augenaffection mit dem Fieber nicht zu verkennen war, über siebenhundert gestiegen. Die Mehrzahl dieser Kranken präsentirte sich ambulatorisch in der hiesigen Augenheilanstalt (sieh. deren Krankenjournale); eine nicht geringe Quote fand ihre Aufnahme im Krankenhause und konnte somit der Verlauf auch clinisch verfolgt werden.

Auf diesem Materiale fussend, komme ich zu den Schlüssen, die meine erste Auffassung des Wesens der Krankheit vollkommen bestätigen und die ich hier bündig zusammenfasse.

*) Compte rendu du Congrès international d'ophthalmologie. Paris 1868. pag. $114-117$. 
Der zu Moskau im Jahre 1865 erschienenen Epidemie der febris recurrens folgte eine Augenaffection nach, mit dem constanten Character einer bestimmten Chorioidealerkrankung, deren characteristische Kennzeichen folgende sind: verschieden geformte und mehr oder weniger ansgesprochene Glaskörperopacitäten bei Abwesenheit solcher objectiven Veränderungen der innern Augenhäute, welche die Entstehung dieser Trübungen zu erklären vermöchten, eine im Laufe der Krankheit hinzutretende, mehr oder weniger intensive Ciliarneurose und meist lebhafte, tiefe Injection der subcon. junctivalen Gefässe um die Cornea, Ausbildung eines kleinen Hypopyon am Grunde der Augenkammer, Verminderung des intraocularen Druckes, secundäre Affection der Iris (bei mässig erweiterter Pupille), des Kammerwassers u. s. w. ${ }^{*}$ )

Diese Chorioidealerkrankung, deren Wesen für eine Ciliarkörperentzündung - cyklitis s. chorioideitis antica - zu halten ist, hat eine grosse Neigung, auf die Iris sich fortzupflanzen und zwar geschieht dieser Uebergang in der Mehrzahl der Fälle, bei Weitem aber nicht immer.**) Dieser Umstand gab und giebt bis jetzt Veranlassung das Wesen des Uebels zu verkennen und zwei verschiedene Krankheitsformen - wie die primäre Entzündung des vordern Abschnitts der Chorioidea und die Iritis mit secundärer Chorioidealerkrankung - mit einander zu verwechseln, - ein Irrthum, den man in der ophthalmologischen Literatur bis auf die letzte Zeit findet.

*) Die Emptindlichkeit, welche sich bei Betastung der Ciliargegend Kund giebt, kamn zu den characteristischen Kennzeichen nicht immer gerechnet werden und fehlt ausserdem in der Mebrzahl der Falle.

**) Unter 266 Fällen war die Iris 56 mal intant, 20 mal kaum verfärbt, 39 mal verfärbt und zeigte Pigmentauflagerungen auf der vordexn Linsenkapsel; 124 mal namhaft verändert, $27 \mathrm{mal}$ starke Iritis. In die zwei letzteren Categorien gehören sehr vieie artificielle Formen. Unter 399 andern Fällen war die Iris 103 mal intact, 30 mal kaum verfärbt und träge. 
Das Bild der in Folge einer febris recurrens sich entwickelnden Ciliarkörperentzündung kann zwei Hauptformen oder Modificationen darstellen. Ob sich die eine oder andere Form entwickeln wird, hängt namentlich von der Intensität des Prozesses ab, welche ihrerseits hauptsächlich durch das Alter des Kranken (auch die Lebensweise bleibt nicht ohne Einfluss) und der damit verbundenen grösseren oder geringern Neigung dieses oder jenes Abschnitts der Uvea zur Erkrankung, bedingt wird. Gewiss besitzt das Kindesalter wenic Neigung zur Iriserkrankung, anderseits stellt der Verlauf der Krankheitsprozesse in diesem Alter einen mehr acuten, lebhaften Character dar. Diese beiden Momente sind auch für unsern Process entscheidend. Einerseits - Symptome einer acuten Entzündung des Ciliarkörpers, respective heftige plötzlich entstehende Gefässinjection um die Cornea, Ciliarneurose, Hypopyon, flockige (eitrige?) Glaskörperopacitäten; andererseits - keine oder entsprechend sehr geringe Betheiligung der Iris an einem ihr so nahe benachbarten Krankheitsprocesse. Diese Form ist besonders geeignet zur Demonstration einer gemeinen acuten Cyklitis.

Die zweite Form trägt an sich das Gepräge der Chronicität, der Asthenie und hat eine viel grössere Neigung auf die Iris überzugreifen. ${ }^{*}$ ) Die äussern Zeichen einer Entzündung sind wenig ausgesprochen, ja sie fehlen sogar. Nie abwesende Glaskörperopacitäten, leichte Verfärbung der Iris, oberflächliche Synechien sind öfters die einzigtn Symptome der Affection. Ein anderes Mal sind es die Erscheinungen einer starken Iritis, die in den Vordergrund treten, so dass man eine selbstständige Iritis vor sich zu haben glaubt, wenn man die

*) In diese Categorie gehören die meisten Fälle der s. g. Iritis serosa mit Glaskörperopacitäten. 
Initialperiode der Krankheit nicht berücksichtigt. Zwischen den zwei Hauptformen werden graduale Uebergangsformen beobachtet. Ausnahmefälle, übrigens ziemlich häufige, bilden reine Glaskörperopacitäten ohne etwaige sichtbare Veränderungen im Auge oder höchstens mit feinen punktförmigen Niederschlägen auf der hintern Oberfläche der Cornea - s. g. Keratitis punctata - gepaart. Es sei noch erwähnt, dass anderweitige Complicationen des in Rede stehenden Processes seitens der Conjunctiva, Sclera, Cornea, Augenlider fasst ausschliesslich von den äussern Schädlichkeiten, respective einer unpassenden Therapie, wozu namentlich die Anwendung aller bis jetzt noch so eifrig verpflegten Augenwässer mit Rosenwasser gerechnet werden muss, herrühren. Im Alter bis vierzehn Jahren sah ich ausschliesslich die erste Form sich ausbilden. Je älter das Subject war, desto seltener stellte es das Bild dieser Form dar, um sodann im Alter nach dreissig Jahren nur von der zweiten Form befallen zu werden.

Die Augenaffection schonte weder schwächliche, heruntergekommene, noch kräftige Subjecte, weder das kindliche, noch das Greisenalter. Von der Gesammtzahl der 715 Fälle kam sie 11mal im Alter von 3 bis 10 Jahren, $174 \mathrm{mal}$ von 10 bis $20,197 \mathrm{mal}$ - bis 30 , $185 \mathrm{mal}$ - bis $40,114 \mathrm{mal}$ - bis 50,29mal bis 60 und $5 \mathrm{mal}$ - bis 70 vor.

Das Alter von 20-40Jahren lieferte somit die grösste Anzahl der Erkrankungen.

730 Fälle vertheilten sich unter 476 Kranke des männlichen und 254 des weiblichen Geschlechts. Auf 142-97mal erkrankten helle Augen (unter ihnen 77 blaue), 45mal dunkle. Das rechte Auge war $310 \mathrm{mal}$ getroffen, das linke 266mal, beide Augen 133mal. Die niedere Classe der Bevölkerung lieferte $94 \mathrm{pCt}$. aller Krankbeitsfälle. 
Um übrigens diese statistischen Angaben zu verwerthen, muss eine parallele Statistik der febris recurrens berücksichtigt werden, es müssen damit statistische Daten über die Vertheilung der Bevölkerung dem Geschlechte nach, über prävalirendes Vorkommen überhaupt, dieser oder jener Färbung der Iris verglichen werden.

Der Augenaffection ging febris recurrens voraus, und zwar in einem Zeitraum von wenigen Tagen bis zu 6 Monaten und selbst darüber; in der Mehrzahl von 2 bis 8 Wochen. Es gab Fälle, wo die Augenaffection im Laufe der Reconvalescenz auftrat, -dagegen ist mir kein einziger Fall bekannt, wo das Auge in der Zwischenzeit der Anfälle erkrankte, somit ist das Leiden als eine wahre Nachkrankheit anzusehen. Seine innige Beziehung zum Fieber wird aus dem Umstande klar, dass sie zum drittenmal epidemisch gleichzeitig mit der Epidemie der febris recurrens auftritt, dass sie diejenige Kranken befällt, welche das genaunte Fieber überstanden haben; endlich erbellt diese Beziehung aus dem Parallelismus der Schwankungen im Gange beider Epidemien. Ich muss übrigens bekennen, dass ich durch meine Beobachtungen keineswegs vor Täuschung, als sei die nämliche Augenaffection nur als Folgezustand der febris recurrens anzusehen, wie es die englischen Aerzte und Blessig thun, absolut gesichert bin. Es kam mir sehr häufig vor, dass ich aus der Anamnese überhaupt blos ein Fieber als Präcedent der Augenaffection annehmen durfte, ohne dasselbe näher präcisiren zu können, und mich in solchen Fällen nur durch Analogie verleiten liess. Es kam mir ja sogar exquisite, reine Cyklitis zweimal nach Fleckentyphus, zweimal nach biliösem Typhoid, einmal nach unregelmässigem Wechselfieber und zweimal nach Blattern vor, (noch eines dritten identischen Falles, der als Iritis serosa mit Glaskörpertrübungen diagnosticirt 
wurde, kann ich mich aus dem Jahre 1862 aus der v. Graefe'schen Klinik erinnern). ${ }^{*}$ ) Jedoch ist die Zahl solcher Fälle verschwindend klein, und sind auch diese nicht von dem Vorwurfe vollkommen frei, als handelte es sich hier um eine zweifelhafte oder irrthümliche Diagnose des Fieberzustandes. Das biliöse Typhoid wird übrigens von den meisten Autoritäten als eine umgestaltete febris recurrens angesehen.

Der Verlauf der Augenaffection ist meistens chronisch. Es vergehen Wochen, Monate, ausnahmsweise sogar Jahre, bevor sie zum Abschluss kommt. Am hartnäckigsten erweisen sich diejenigen Veränderungen, mit denen die Krankheit debütirt, d. h. die Glaskörperopacitäten. Eine vollkommene Genesung ist der häufigste Ausgang. Daneben sind Fälle zu notiren, wo eine leichte Amblyopie, die mit vermuthlich bleibenden, oft unsichtbaren Veränderungen in der Glaskörperstructur in Zusammenhang zu bringen ist, sich ausbildet. Ferner wird die Amblyopie durch hintere Polarcataract, bleibende Glaskörpertrübungen, aequatoriale Veränderungen im Pigmentepithel der Chorioidea (der Retina?) bedingt. Wo jedoch die Krankheit zu sehr vernachlässigt wird oder, noch schlimmer, durch eine unzweckmässige Therapie behandelt, da findet man öfters hintere Synechien, selbst Pupillarverschluss mit seinen übeln Folgen und Netzhautablösung. Dieser letzte Ausgang ist mir im Ganzen nur zweimal vorgekommen und führte er in einem Falle den unglücklichen Kranken, einen Bauernknaben, zur völligen Blindheit, da fast totale Netzhautablösung doppelseitig sich ausbildete. Niemals sah ich dagegen Glaucom, sympathische Entzündung, oder Pthisis bulbi entstehen.

*) Der Kranke, ein Handwerker, wurde am 1. December 1862 vorgestellt und entzog sich am 18. December der weiteren Beobachtung. 
Die Therapie war höchst einfach, In leichten Fällen reichte man mit Atropin vollkommen aus. Gegen starke entzündliche Symptome wandte man den künstlichen Biutegel, Mercurialien, in späteren Perioden Kalium jodatum, oder je nach Umständen roborirende Mittel an.

Unabbängig von den oben erwähnten Symptomen einer Cyklitis, schien es mir wichtig, ein Kennzeichen zu finden, das beim allerersten Beginne der Affection auf die primäre Localisation derselben im vordern $A b$ schnitte der Aderhaut, im Ciliarkörper, hinweisen könnte.

Ein solches Symptom konnte natürlich nur in der anatomischen Structur oder physiologischen Wirkung des Ciliarkörpers selbst, gesucht werden.

Bei der Unmöglichkeit, dessen Veränderungen bei Lebzeiten zur unmittelbaren Anschauung zu bringen, hätte man eine pathologisch-anatomische Untersuchung abwarten müssen, zu der aber bis jetzt sich keine Gelegenheit darbot. Ich musste mich also zu functionellen Erscheinungen wenden, und dazu Krankheilsfälle in der allerersten Periode, wo also bei vorkommender Integrität der Iris noch keine äusseren Zeichen und höchtens beginnende diffuse Glaskörpertrübungen vorhanden waren, aufsuchen, und konnte ich in sechs solchen Fällen, wo ich mit vernünftigen und schriftkundigen Patienten zu thun hatte, fünfmal eine nicht zu verkennende Accommodationsparese constatiren. Ausserdem war allen sechs jede Accommodationsanstrengung höchst unangenehm und lästig.

So viel über die im Zusammenhange mit febris recurrens vorkommende Entzündung des Ciliarkörpers.

Sie erlauben mir, meine Herren, noch einige Bemerkungen zur Pathogenese der primären Cyklitis überhaupt. Die Krankheit hat keineswegs das Privilegium blos als Folgezustand der febris recurrens aufzutreten, vielmehr kommt sie auch selbständig yor, muss aber in 
diesem Falle als eine seltene Erscheinung in der Reihe anderer Augenkrankheiten angesehen werden. Wenigstens in Moskau macht sie kaum 0,5 pro mille aller Augenkrankheiten aus. Häufiger tritt sie in Folge einer Trauma der Ciliargegend durch eingedrungene fremde Körper (Metallsplitter), blähende oder vormals reclinirte Linse u. dgl. auf und wird sie in solchen Fällen meistens zum Ursprung einer deletären Augenentzündung.

Was das Geschichtliche anbelangt, so war kaum irgend eine andere Augenkrankheit in ihrem Wesen so oft verkannt, bis auf die letzte Zeit so sehr vernachlässigt. In der älteren Literatur findet ihre Beschreibung regelmässig Platz unter den Namen: sclerotitis, retinitis, ophthalmia rheumatica, ophthalmodes mitis, aquocapsulitis, keratoiritis u. s. w. Hingegen liest man unter dem Titel der Cyklitis, Entzündung des orbiculus ciliaris, chorioiditis am allerwenigsten die Schilderung der in Rede stehenden Augenkrankheit. Die neuere Literatur behandelte die Cyklitis nicht weniger stiefmütterlich und wurde die letzte mit iritis, iritis serosa, s. g. keratitis punctata, iridochorioideitis, ophthalmia sympathica, hyaloitis zusammengeworfen.*) Auch in diesem Gebiete der Ophthalmologie, wie in zahllosen anderen, gebührt $v$. Graefe die Ehre, zuerst die reine Cyklitis erkannt und eine kurze, aber nichts desto weniger werthvolle Skizze derselben gegeben zu haben.**) In einer dieser Skizzen möchte ich nur das beständige Vorkommen der Glaskörpertrübungen für die primäre Cyklitis viel nachdrücklicher hervorgehoben sehen.

*) Eine gIückliche Ausnahme findet man im Handbuch der Augenheilkunde von W. Zehender, Lief. III. p. 676-678, wo Cyklitis, obgleich mit Reserve, ihr Bürgerrecht erlangt. Ihre Charakteristik kann übrigens nicht zu den gelungensten gerechnet werden.

**) Siehe; Archiv für Ophthalmol. Bd. III. 2, p. 353-354. Vergl. auch $B$ d. II. 2, p. 330 u. folgende. 
Noch ein paar Worte zur Nomenclatur. Die in Rede stehende Augenkrankheit nenne ich cyklitis oder chorioideitis antica (vel anterior) und finde beide Benennungen gerechtfertigt da, wo es sich um eine Entzündung desjenigen Chorioidealabschnittes handelt, welcher sein, im Sinne Leber's*) vom hintern Abschnitte unabhängiges Gefässbezirk aus den hintern langen und vordern Ciliararterien besitzt. In diesem Sinne würde Cyklitis zu Chorioideitis antica sich etwa wie ein Theil (und zwar der grösste und wesentlichste) zu seinem Ganzen verhalțen. Die chorioideitis äquatorialis (v. Graefe) möchte ich von chorioideitis antica trennen, da die letzte nur diesseits des Aequators zu liegen kommt und hierselbst ihre Begrenzung findet. - Eine ansführliche Arbeit über den Gegenstand wird in Kurzem separat erscheinen.

$$
\text { Moska u, } \frac{28 . \text { Aug. }}{9 \text {. Sept. }} 1869 \text {. }
$$

*) Siehe: Th. Leber. Untersuchungen über den Verlauf und Zu-sammenhang der Gefässe im menschlichen Auge. Arch. f. Ophthalmologie XI. 1, p. 18 und 19. 